\title{
Polyphasic characterisation of Burkholderia cepacia complex species isolated from children with cystic fibrosis
}

\author{
Fernando José Vicenzi ${ }^{1,2}$, Marcelo Pillonetto ${ }^{3,4}$, \\ Helena Aguilar Peres Homem de Mello de Souza ${ }^{5}$, Jussara Kasuko Palmeiro, ${ }^{2,5}$, \\ Carlos Antônio Riedi ${ }^{6}$, Nelson Augusto Rosario-Filho ${ }^{6}$, Libera Maria Dalla-Costa ${ }^{2,5} /+$
}

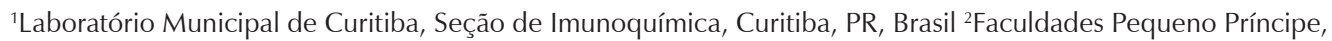
Instituto de Pesquisa Pelé Pequeno Principe, Curitiba, PR, Brasil ${ }^{3}$ Pontifícia Universidade Católica do Paraná, Escola de Saúde e Biociências, Curso de Farmácia, Departamento de Microbiologia, Curitiba, PR, Brasil ${ }^{4}$ Laboratório Central do Estado do Paraná, Seção de Biologia Molecular, Curitiba, PR, Brasil ${ }^{5}$ Universidade Federal do Paraná, Faculdade de Medicina, Hospital de Clínicas, Seção de Bacteriologia, Curitiba, PR, Brasil ${ }^{6}$ Universidade Federal do Paraná, Faculdade de Medicina, Departamento de Pediatria, Curitiba, PR, Brasil

Cystic fibrosis (CF) patients with Burkholderia cepacia complex (Bcc) pulmonary infections have high morbidity and mortality. The aim of this study was to compare different methods for identification of Bcc species isolated from paediatric CF patients. Oropharyngeal swabs from children with CF were used to obtain isolates of Bcc samples to evaluate six different tests for strain identification. Conventional (CPT) and automatised (APT) phenotypic tests, polymerase chain reaction (PCR)-recA, restriction fragment length polymorphism-recA, recA sequencing, and matrix-assisted laser desorption ionization-time of flight (MALDI-TOF) were applied. Bacterial isolates were also tested for antimicrobial susceptibility. PCR-recA analysis showed that 36 out of the 54 isolates were Bcc. Kappa index data indicated almost perfect agreement between $C P T$ and APT, CPT and PCR-recA, and APT and PCR-recA to identify Bcc, and MALDI-TOF and recA sequencing to identify Bcc species. The recA sequencing data and the MALDI-TOF data agreed in $97.2 \%$ of the isolates. Based on recA sequencing, the most common species identified were Burkholderia cenocepacia IIIA (33.4\%), Burkholderia vietnamiensis $(30.6 \%)$, B. cenocepacia IIIB (27.8\%), Burkholderia multivorans (5.5\%), and B. cepacia (2.7\%). MALDI-TOF proved to be a useful tool for identification of Bcc species obtained from CF patients, although it was not able to identify B. cenocepacia subtypes.

Key words: Burkholderia cepacia complex - cystic fibrosis - lung disease - Gram-negative bacterial infection DNA sequence analyses - matrix-assisted laser desorption-ionisation mass spectrometry

Cystic fibrosis (CF) pathophysiology leads to an increased susceptibility to respiratory infections (Drevinek \& Mahenthiralingam 2010). The Burkholderia cepacia complex (Bcc) prevalence rates in CF patients vary geographically and have been shown to be $1.8 \%$ in Belgium (De Boeck et al. 2004) and 5\% in Canada (CFC 2015); in Brazilian children, the prevalence was 4\% (Souza et al. 2006). CF patients with Bcc infection have high rates of morbidity and mortality (Drevinek \& Mahenthiralingam 2010, LiPuma 2010). Bcc infections pose potential for patient-to-patient transmission, which can lead to the development of "cepacia syndrome," a fatal necrotising pneumonia (Drevinek \& Mahenthiralingam 2010).

Currently, Bcc includes 20 species; however, new species have been identified or reclassified over the years (Drevinek \& Mahenthiralingam 2010, LiPuma 2010, Vandamme \& Dawyndt 2011, Peeters et al. 2013, Smet et al. 2015). Burkholderia multivorans and Burkhol-

doi: $10.1590 / 0074-02760150314$

+ Corresponding author: libera.costa@hc.ufpr.br

Received 21 August 2015

Accepted 1 December 2015 deria cenocepacia were shown to be the prevalent species (Drevinek \& Mahenthiralingam 2010, LiPuma 2010). Correct species identification of Bcc is essential for treating CF patients (Gilligan et al. 2006) because of interspecies variation in antimicrobial susceptibility (Leitão et al. 2008) and potential for patient-to-patient transmission (Drevinek \& Mahenthiralingam 2010). However, accurate Bcc identification is challenging due to the phenotypic and genetic similarities among species.

Bcc species comprise phenotypically indistinguishable microorganisms with high genetic similarity (> 97.5\%) that was determined using 16S rDNA sequencing (Coenye et al. 2001, Vandamme \& Dawyndt 2011). Conversely, 16S rDNA sequencing assays cannot accurately discriminate among $B$. cepacia, $B$. cenocepacia, Burkholderia ambifaria, Burkholderia pyrrocinia, and Burkholderia anthina (Vermis et al. 2002b). Furthermore, $r e c A$ sequencing data showed that the Bcc interspecies and intraspecies similarities were $94-95 \%$ and $98-99 \%$, respectively (Vanlaere et al. 2008, Vandamme \& Dawyndt 2011). Thus, Bcc inter and intraspecies discrimination is challenging.

The discriminatory power of $r e c A$ sequencing method is better than $16 \mathrm{~S}$ rDNA sequencing analysis because $r e c A$ has enough nucleotide variability to enable differentiation of the main Bcc species isolated from CF patients (Mahenthiralingam et al. 2000). 
Selective media culture and several methods, including phenotypic methods, conventional and automated methods, have been used for Bcc identification. However, reliable and accurate identification of Bcc requires the use of molecular assays such as polymerase chain reaction (PCR)-based assays, PCR-restriction fragment length polymorphism (RFLP-PCR), DNA sequencing, and matrix-assisted laser desorption ionization-time of flight (MALDI-TOF). A study comparing the proficiency of these tests would be valuable for developing a multi-method approach with higher accuracy.

The aim of this study was to compare different methods for identification of Bcc species isolated from oropharyngeal swabs of paediatric CF patients.

\section{SUBJECTS, MATERIALS AND METHODS}

Bacterial samples - Oropharyngeal swabs were collected from 46 CF patients (aged 2-90 months) attended at the Cystic Fibrosis Outpatient Clinic of the Department of Paediatrics of the Federal University of Paraná (UFPR) Clinics Hospital from August 2003-February 2009 (Souza et al. 2006, Souza 2012) as previously described (Souza et al. 2006).

Fifty-four bacteria samples grown in B. cepacia selective agar (Henry et al. 1997), presumptively identified as Bcc by polymyxin B susceptibility, oxidase test, and lysine decarboxylation test, were included in the study. Bacteria isolates were kept at $-80^{\circ} \mathrm{C}$ until subjected to the methods studied.

Phenotypic identification - Fifty-four bacterial isolates were subjected to conventional phenotypic tests (CPT) and automated phenotypic tests (APT). CPT was performed as previously described (Schreckenberger et al. 2003). APT identification was carried out using GN ID cards in the Vitek $^{\circledR} 2$ Compact system (bioMérieux, USA).

Susceptibility testing - Minimum inhibitory concentrations (MICs) of ceftazidime (CAZ), meropenem (MER), levofloxacin (LEV), and sulfamethoxazole/ trimethoprim (SUT) were performed (in 36 bacterial isolates) using the agar dilution test protocol described in Clinical and Laboratory Standards Institute (CLSI) M07-A09 (CLSI 2012) and by using interpretive criteria described in CLSI M100-S24 (CLSI 2014). Pseudomonas aeruginosa ATCC 27853 and Escherichia coli ATCC 25922 were used as control strains.

Extraction, purification, and quantification of bacterial DNA - Template DNA of the 54 bacterial isolates was obtained using post-boiling extraction technique (Navrátilová et al. 2013) for performing recA PCR amplification and RFLP-PCR (Mahenthiralingam et al. 2000). AccuPrep Genomic DNA Extraction kit (Bioneer Corporation, Korea) were used for the 36 bacterial isolates subject to recA sequencing. The DNA concentrations of samples were quantified using Nano Vue Plus (GE Healthcare Bio-Sciences Corp, USA) and adjusted to concentrations between $20-50 \mathrm{ng} / \mu \mathrm{L}$.

PCR for recA - Primers BCR1 (5' $\rightarrow 3^{\prime}$ TGAGCCGCCGCAAGAAGAA) and BCR2 $\left(5^{\prime} \rightarrow 3^{\prime}\right.$ CTCTTCCATTTCGTCCTCCGC) (Mahenthiralingam et al. 2000) were used for the identification of Bcc. PCR mixture (50 $\mu \mathrm{L})$ consisted of $1.5 \mathrm{mM} \mathrm{MgCl}, 0.4 \mu \mathrm{M}$ of each primer, $0.2 \mathrm{mM}$ each deoxynucleoside triphosphate, $0.02 \mathrm{U} /$ $\mu \mathrm{L}$ Taq DNA polymerase, $1 \times$ PCR buffer, $5 \mu \mathrm{L}$ template DNA (20-50 ng/ $\mathrm{LL}$ ), and $35.5 \mu \mathrm{L}$ of PCR-grade water. Amplification was performed using Techne TC-412 thermal cycler (Bibby Scientific Ltd, UK) under the following conditions: 1 cycle at $95^{\circ} \mathrm{C}$ for 5 min followed by 35 cycles at $95^{\circ} \mathrm{C}$ for $30 \mathrm{~s}, 65^{\circ} \mathrm{C}$ for $45 \mathrm{~s}$, and $72^{\circ} \mathrm{C}$ for 60 $\mathrm{s}$. The final extension was performed using 1 cycle at $72^{\circ} \mathrm{C}$ for $10 \mathrm{~min}$. B. cepacia ATCC 17759 and P. aeruginosa ATCC 27853 were used as positive and negative controls, respectively. The Bcc-positive were confirmed by the presence of a band of approximately $1,040 \mathrm{bp}$.

PCR-RFLP for recA - Amplicons obtained with BCR1 and BCR2 primers were digested with HaeIII enzyme and each agarose gel (1.5\%) electrophoresis profile was compared to those of the following control strains: B. cenocepacia IIIA, B. cenocepacia IIIB, Burkholderia stabilis, B. multivorans, B. cepacia, and Burkholderia vietnamiensis to identify the Bcc species (Mahenthiralingam et al. 2000).

recA sequencing - The recA amplicons were purified with ExoSAP-IT (Affymetrix, USA) and then sequenced using BigDye Terminator v.3.1 Cycle Sequencing Kit (Applied Biosystems, USA). The 1,043 bp recA gene was sequenced in two fragments of approximately $500 \mathrm{bp}$ using a combination of primers BCR1 $(5$ ' $\rightarrow 3$ 'TGAGCCGCCGCAAGAAGAA) with BCR4 $\left(5^{\prime} \rightarrow 3^{\prime}\right.$ GCGCAGCGCCTGCGACAT) and BCR2 $\left(5^{\prime} \rightarrow 3^{\prime}\right.$ CTCTTCCATTTCGTCCGCCTC) with BCR3 $\left(5^{\prime} \rightarrow 3^{\prime}\right.$ GTCGCAGGCGCTGCGCAA) (Mahenthiralingam et al. 2000). The amplicons were purified using BigDye sequencing X Terminator Purification Kit (Applied Biosystems, Life Technologies Corporation) and sequenced. Each sample generated four sequences that were analysed by using Bioedit software (mbio. ncsu.edu/bioedit/bioedit.html). The obtained consensus sequence was then submitted to PubMLST Bcc allele recA database (pubmlst.org/bcc/).

MALDI-TOF analysis - MALDI-TOF protein extraction was performed as previously described (Khot et al. 2012). The extract was inoculated in duplicate, air-dried, and covered with $1 \mathrm{~mL}$ saturated $\alpha$-cyano-4-hydroxycinnamic acid in $50 \%$ acetonitrile and $2.5 \%$ trifluoroacetic acid solution. Mass spectra were determined by using Bruker Daltonics Microflex LT instrument with MALDI Biotyper 3.0 software (Bruker Daltonics, Germany), previously calibrated with Bruker Bacterial Test Standard (Escherichia coli DH5 $\alpha$ ) and the mass spectra were generated using a mass range of $2-\mathrm{kDa}$ obtained by ionisation of 240 shots. Species identification and genus were obtained when the scores were $\geq 2.0$ and between $\geq 1.7$ and $<2.0$, respectively, as recommended by the manufacturer. B. cepacia ATCC 25608, $P$. aeruginosa ATCC 27853, and E. coli ATCC 25922 were used as controls.

Statistical analyses - The analysis of agreement between the methods was performed using the kappa (k) test with criteria outlined by Landys \& Koch (1977): 
poor agreement $(\mathrm{k}<0)$, slight agreement $(\mathrm{k}$ : 0.01-0.2), reasonable agreement $(\mathrm{k}: 0.21-0.40)$, moderate agreement (k: 0.41-0.60), substantial agreement (k: 0.61-0.80), and almost perfect agreement (k: 0.81-1.00). APT and CPT proficiency analyses were performed using contingency table or $2 \times 2$ matrix.

Ethics - The study design and the consent form were approved by the Clinics Hospital/UFPR Institutional Ethical Review Board.

\section{RESULTS}

Thirty-six out of the fifty-four preliminarily identified Bcc strains were confirmed as Bcc by using recA PCR amplification. The discordancy in Bcc identification was highlighted by comparative analysis of the findings of the five methodologies (CPT, APT, recA PCR, recA sequencing, and MALDI-TOF) (Table I). Notably, identification of one bacterial isolate did not agree by different techniques: Bordetella spp by CPT, Cupriavidus pauculus by APT, and as $B$. cepacia by both rec $A$ sequencing method and MALDI-TOF. One strain of $B$. vietnamiensis could not be conclusively identified by using CPT (Table I).

The recA sequencing data and MALDI-TOF data, for the majority of the 36 strains, were in agreement and corroborated with Bcc identification data. However, one strain identified as $B$. vietnamiensis by recA sequencing was identified as $B$. cenocepacia by MALDI-TOF (Table I).

The identification data obtained by the aforementioned methods were used for performing comparative analyses to assess whether Bcc or Bcc genomic variants were identified consistently. The concordance between phenotypic test data (CPT vs. APT) and that among different tests was also assessed (Table II).

RecA amplification data showed almost perfect agreement with CPT data (k: 0.833) and with APT data (k: 0.921). In addition, MALDI-TOF data was also in almost perfect agreement with $\operatorname{rec} A$ sequencing data $(\mathrm{k}$ : 0.942). However, PCR-RFLP and $r e c A$ sequencing data presented moderate agreement (k: 0.592). The phenotypic data and the recA amplification data were also compared. CPT and APT had assay specificity of $94.44 \%$ and $97.62 \%$, respectively (Table III). Furthermore, the assay sensitivity of CPT and APT were $81.25 \%$ and $100 \%$, respectively (Table III).

PCR-RFLP analysis of 36 isolates resulted in nine different restriction profiles. Three different patterns $(B, C$, and F) were obtained for $B$. cenocepacia IIIB and one for B. cenocepacia IIIA (pattern A). Pattern D was associated with $B$. multivorans. The pattern E was linked with $B$. cepacia. Two patterns (H and I) were related to B. vietnamiensis. The pattern $\mathrm{G}$ corresponded to $\mathrm{C} 12$ B. stabilis control.

Our data showed prevalence rate of $33.4 \%$ (12/36) for B. cenocepacia IIIA, 30.6\% (11/36) for B. vietnamiensis, $27.8 \%$ (10/36) for $B$. cenocepacia IIIB, 5.5\% (2/36) for $B$. multivorans, and $2.7 \%$ (1/36) for B. cepacia.

Antimicrobial susceptibility testing data presented as follows: the B. cepacia isolate was susceptible to all antimicrobial agents tested; all $B$. cenocepacia $(22 / 22)$ were susceptible to CAZ, LEV, and MER, and $68.2 \%(15 / 22)$ were susceptible to SUT; all B. multi- vorans (2/2) showed sensitivity to LEV, CAZ, and MER, but presented resistance to SUT; all $B$. vietnamiensis $(11 / 11)$ were sensitive to LEV, $81.8 \%(9 / 11)$ B. vietnamiensis were sensitive to CAZ, $90.9 \%(10 / 11)$ B. vietnamiensis isolates were sensitive to MER, and $72.7 \%(8 / 11)$ $B$. vietnamiensis isolates were sensitive to SUT.

\section{DISCUSSION}

Owing to the similarity in phenotypic characteristics of Bcc species, interspecies distinction is challenging (Vandamme \& Dawyndt 2011). A comparative analysis between CPT and APT was performed using $\mathrm{k}$ test and the results indicated that the findings were in agreement; however, it is important to note that the quality of the CPT results depends on the experience and expertise of the microbiologist. A comparative analysis of phenotypic test data and recA amplification data indicated that CPT and APT data were in agreement with recA amplification data; however, the positive predictive value, specificity, and the k coefficient of APT was higher than that of CPT. Thus, APT may be a better test than CPT for Bcc identification. Although APT cannot adequately perform Bcc interspecies distinction, it could be used for Bcc identification in laboratories lacking molecular biology facilities.

MALDI-TOF proved to be a useful tool for identification of Bcc species obtained from CF patients, although it was not able to identify B. cenocepacia subtypes. This finding corroborated those from other studies (Bittar \& Rolain 2010, Fehlberg et al. 2013). MALDI-TOF is a rapid and single step assay that does not require personnel with assay expertise. Furthermore, MALDI-TOF and rec $A$ sequencing assays have comparable Bcc identification accuracy (Bittar \& Rolain 2010, Fehlberg et al. 2013).

Studies have shown that $\operatorname{rec} A$ PCR using speciesspecific primers is not sensitive and specific (Dalmastri et al. 2005, Mahenthiralingam et al. 2008, Vandamme \& Dawyndt 2011) and sequencing is required for the final identification (Mahenthiralingam et al. 2002, Vermis et al. 2002a). Comparison between PCR-RFLP and recA sequencing data indicates only moderate correlation between the findings of the two methods. Other studies have shown that HaeIII RFLP patterns insufficiently discriminated B. cepacia, B. cenocepacia, and B. stabilis (Vanlaere et al. 2009, Navrátilová et al. 2013). Previous studies demonstrated the limited usefulness of PCR-RFLP technique for discriminating Bcc: the same variants may have different genomic restriction patterns, one restriction pattern can be associated with different species, and interlaboratory reproducibility is impaired because more than 70 recA-HaeIII restriction patterns have been identified. In addition, the requirement of a standard control for each species and its subtypes become the precise identification of each isolate not practical (Vanlaere et al. 2008, Vandamme \& Dawyndt 2011). Although the results suggest the optimal performance of MALDI-TOF of Bcc species identification, some studies indicate a certain difficulty of this method to differentiate some strains of B. cepacia and B. cenocepacia (Vandamme \& Dawyndt 2011, Navrátilová et al. 2013).

This study found the highest prevalence rates for $B$. cenocepacia and this finding was similar to those re- 
TABLE I

Burkholderia cepacia complex (Bcc) identification data obtained by using different methodologies

\begin{tabular}{|c|c|c|c|c|c|c|c|c|}
\hline Patient & Isolate & CPT & APT & $\begin{array}{l}\text { PCR } \\
\operatorname{rec} A\end{array}$ & $\begin{array}{c}\text { Seq.RecA } \\
\text { PubMLST } \\
\text { Bcc database }\end{array}$ & MALDI-TOF & $\begin{array}{l}\text { Allele } \\
\text { recA }\end{array}$ & RFLP \\
\hline \multirow[t]{2}{*}{1} & $\mathrm{BC}-48-\mathrm{FC}$ & Bordetella sp. & $\begin{array}{c}\text { Cupriavidus } \\
\text { pauculus }\end{array}$ & $\mathrm{Bcc}$ & B. cenocepacia IIIB & B. cenocepacia & 15 & $\mathrm{~F}$ \\
\hline & $\mathrm{BC}-5-\mathrm{FC}$ & $\mathrm{Bcc}$ & $\mathrm{Bcc}$ & $\mathrm{Bcc}$ & B. cepacia & B. cepacia & 37 & $\mathrm{E}$ \\
\hline \multirow[t]{2}{*}{2} & $\mathrm{BC}-28-\mathrm{FC}$ & $\mathrm{Bcc}$ & $\mathrm{Bcc}$ & $\mathrm{Bcc}$ & B. cenocepacia IIIB & B. cenocepacia & $15^{a}$ & $\mathrm{~F}$ \\
\hline & BC-54-FC & $\mathrm{Bcc}$ & $\mathrm{Bcc}$ & $\mathrm{Bcc}$ & B. cenocepacia IIIB & B. cenocepacia & $15^{a}$ & $\mathrm{~F}$ \\
\hline 3 & $\mathrm{BC}-25-\mathrm{FC}$ & $\mathrm{Bcc}$ & $\mathrm{Bcc}$ & $\mathrm{Bcc}$ & B. cenocepacia IIIA & B. cenocepacia & $14^{a}$ & A \\
\hline 4 & BC-32-FC & $\mathrm{Bcc}$ & $\mathrm{Bcc}$ & $\mathrm{Bcc}$ & B. cenocepacia IIIA & B. cenocepacia & $14^{a}$ & A \\
\hline 5 & $\mathrm{BC}-46-\mathrm{FC}$ & $\mathrm{Bcc}$ & $\mathrm{Bcc}$ & $\mathrm{Bcc}$ & B. cenocepacia IIIB & B. сепосерасіа & $15^{a}$ & $\mathrm{C}$ \\
\hline \multirow[t]{2}{*}{6} & BC-10-FC & $\mathrm{Bcc}$ & $\mathrm{Bcc}$ & $\mathrm{Bcc}$ & B. cenocepacia IIIA & B. cenocepacia & 14 & A \\
\hline & BC-15-FC & $\mathrm{Bcc}$ & $\mathrm{Bcc}$ & $\mathrm{Bcc}$ & B. cenocepacia IIIA & B. cenocepacia & $14^{a}$ & A \\
\hline \multirow[t]{2}{*}{7} & BC-1-FC & $\mathrm{Bcc}$ & $\mathrm{Bcc}$ & $\mathrm{Bcc}$ & B. multivorans & B. multivorans & $81^{a}$ & $\mathrm{D}$ \\
\hline & BC-20-FC & $\mathrm{Bcc}$ & $\mathrm{Bcc}$ & $\mathrm{Bcc}$ & B. multivorans & B. multivorans & $81^{a}$ & $\mathrm{D}$ \\
\hline \multirow[t]{9}{*}{8} & $\mathrm{BC}-11-\mathrm{FC}$ & $\mathrm{Bcc}$ & $\mathrm{Bcc}$ & $\mathrm{Bcc}$ & B. vietnamiensis & B. cenocepacia & $23^{a}$ & $\mathrm{H}$ \\
\hline & BC-18-FC & $\mathrm{Bcc}$ & $\mathrm{Bcc}$ & $\mathrm{Bcc}$ & B. vietnamiensis & B. vietnamiensis & $23^{a}$ & $\mathrm{H}$ \\
\hline & BC-19-FC & $\mathrm{Bcc}$ & $\mathrm{Bcc}$ & $\mathrm{Bcc}$ & B. vietnamiensis & B. vietnamiensis & 23 & $\mathrm{H}$ \\
\hline & $\mathrm{BC}-21-\mathrm{FC}$ & $\mathrm{Bcc}$ & $\mathrm{Bcc}$ & $\mathrm{Bcc}$ & B. vietnamiensis & B. vietnamiensis & $23^{a}$ & $\mathrm{H}$ \\
\hline & BC-29-FC & $\mathrm{Bcc}$ & $\mathrm{Bcc}$ & $\mathrm{Bcc}$ & B. vietnamiensis & B. vietnamiensis & $23^{a}$ & $\mathrm{H}$ \\
\hline & $\mathrm{BC}-42-\mathrm{FC}$ & Inconclusive & $\mathrm{Bcc}$ & $\mathrm{Bcc}$ & B. vietnamiensis & B. vietnamiensis & $23^{a}$ & $\mathrm{H}$ \\
\hline & BC-58-FC & $\mathrm{Bcc}$ & $\mathrm{Bcc}$ & $\mathrm{Bcc}$ & B. vietnamiensis & B. vietnamiensis & $23^{a}$ & $\mathrm{H}$ \\
\hline & BC-59-FC & $\mathrm{Bcc}$ & $\mathrm{Bcc}$ & $\mathrm{Bcc}$ & B. vietnamiensis & B. vietnamiensis & $23^{a}$ & $\mathrm{H}$ \\
\hline & BC-7-FC & $\mathrm{Bcc}$ & $\mathrm{Bcc}$ & $\mathrm{Bcc}$ & B. vietnamiensis & B. vietnamiensis & $23^{a}$ & $\mathrm{H}$ \\
\hline 9 & BC-27-FC & $\mathrm{Bcc}$ & $\mathrm{Bcc}$ & $\mathrm{Bcc}$ & B. cenocepacia IIIB & B. cenocepacia & $15^{a}$ & $\mathrm{~F}$ \\
\hline \multirow[t]{2}{*}{10} & $\mathrm{BC}-33-\mathrm{FC}$ & $\mathrm{Bcc}$ & $\mathrm{Bcc}$ & $\mathrm{Bcc}$ & B. cenocepacia IIIA & B. cenocepacia & $14^{a}$ & A \\
\hline & $\mathrm{BC}-3-\mathrm{FC}$ & $\mathrm{Bcc}$ & $\mathrm{Bcc}$ & $\mathrm{Bcc}$ & B. cenocepacia IIIA & B. cenocepacia & $14^{a}$ & A \\
\hline \multirow[t]{4}{*}{11} & $\mathrm{BC}-36-\mathrm{FC}$ & $\mathrm{Bcc}$ & $\mathrm{Bcc}$ & $\mathrm{Bcc}$ & B. cenocepacia IIIA & B. cenocepacia & $14^{a}$ & A \\
\hline & BC-56-FC & $\mathrm{Bcc}$ & $\mathrm{Bcc}$ & $\mathrm{Bcc}$ & B. cenocepacia IIIB & B. cenocepacia & $15^{a}$ & $\mathrm{C}$ \\
\hline & BC-60-FC & $\mathrm{Bcc}$ & $\mathrm{Bcc}$ & $\mathrm{Bcc}$ & B. cenocepacia IIIB & B. cenocepacia & $15^{a}$ & $\mathrm{C}$ \\
\hline & BC-61-FC & $\mathrm{Bcc}$ & $\mathrm{Bcc}$ & $\mathrm{Bcc}$ & B. cenocepacia IIIB & B. cenocepacia & 122 & $\mathrm{C}$ \\
\hline 12 & $\mathrm{BC}-2-\mathrm{FC}$ & $\mathrm{Bcc}$ & $\mathrm{Bcc}$ & $\mathrm{Bcc}$ & B. vietnamiensis & B. vietnamiensis & $48^{a}$ & I \\
\hline 13 & $\mathrm{BC}-50-\mathrm{FC}$ & $\mathrm{Bcc}$ & $\mathrm{Bcc}$ & $\mathrm{Bcc}$ & B. vietnamiensis & B. vietnamiensis & $48^{a}$ & I \\
\hline 14 & $\mathrm{BC}-22-\mathrm{FC}$ & $\mathrm{Bcc}$ & $\mathrm{Bcc}$ & $\mathrm{Bcc}$ & B. cenocepacia IIIB & B. cenocepacia & $15^{a}$ & $\mathrm{~F}$ \\
\hline \multirow[t]{5}{*}{15} & BC-34-FC & $\mathrm{Bcc}$ & $\mathrm{Bcc}$ & $\mathrm{Bcc}$ & B. cenocepacia IIIA & B. cenocepacia & $14^{a}$ & A \\
\hline & BC-38-FC & $\mathrm{Bcc}$ & $\mathrm{Bcc}$ & $\mathrm{Bcc}$ & B. cenocepacia IIIA & B. cenocepacia & $14^{a}$ & A \\
\hline & BC-39-FC & $\mathrm{Bcc}$ & $\mathrm{Bcc}$ & $\mathrm{Bcc}$ & B. cenocepacia IIIA & B. cenocepacia & $14^{a}$ & A \\
\hline & $\mathrm{BC}-41-\mathrm{FC}$ & $\mathrm{Bcc}$ & $\mathrm{Bcc}$ & $\mathrm{Bcc}$ & B. cenocepacia IIIA & B. cenocepacia & $14^{a}$ & A \\
\hline & $\mathrm{BC}-57-\mathrm{FC}$ & $\mathrm{Bcc}$ & $\mathrm{Bcc}$ & $\mathrm{Bcc}$ & B. cenocepacia IIIA & B. cenocepacia & $14^{a}$ & A \\
\hline 16 & BC-52-FC & $\mathrm{Bcc}$ & $\mathrm{Bcc}$ & $\mathrm{Bcc}$ & B. cenocepacia IIIB & B. сепосерасіа & $49^{a}$ & $\mathrm{~B}$ \\
\hline
\end{tabular}

a: exact recA allele match; APT: automated phenotypic tests; CPT: conventional phenotypic tests; MALDI-TOF: matrix-assisted laser desorption ionization-time of flight; PCR: polymerase chain reaction; RFLP: restriction fragment length polymorphism.

ported in epidemiological studies of patients with CF and Bcc (Mahenthiralingam et al. 2002, Drevinek \& Mahenthiralingam 2010, LiPuma 2010). The prevalence of $B$. cenocepacia IIIA determined in our study was similar to that reported in Canada, UK, Italy, and other European countries, while IIIB is prevalent in United States of America (USA) (Mahenthiralingam et al. 2002). However, higher $B$. multivorans prevalence in $\mathrm{CF}$ centres in the UK and the USA has been recently reported and the increased prevalence may be due to the approaches taken in these centres to control B. cenocepacia outbreaks (Mahenthiralingam et al. 2008, Drevinek \& Mahenthiralingam 2010, LiPuma 2010). B. vietnamiensis was identified as the second most prevalent genomic variant and these findings were in agreement with those of a previous study (LiPuma et al. 2001). 
TABLE II

Evaluation of agreement among methods

\begin{tabular}{|c|c|c|c|}
\hline Tests (n) ${ }^{a}$ & $\mathrm{k}$ & SE & Agreement $^{b}$ \\
\hline CPT vs. APT (54) & 0.833 & 0.080 & Almost perfect agreement \\
\hline CPT vs. rec $A \mathrm{PCR}^{c}(54)$ & 0.833 & 0.080 & Almost perfect agreement \\
\hline APT vs. recA PCR (54) & 0.921 & 0.055 & Almost perfect agreement \\
\hline MALDI-TOF vs. recA $\mathrm{Seq}^{d}(36)$ & 0.942 & 0.057 & Almost perfect agreement \\
\hline RFLP $^{e}$ vs. $r e c A$ Seq (36) & 0.592 & 0.099 & Moderate agreement \\
\hline
\end{tabular}

$a$ : number of isolates tested by each test; $b$ : adapted interpretation criteria (Landis \& Koch 1977); $c$ amplification of recA; $d$ : sequencing of recA; $e$ : HaeIII-restriction fragment length polymorphism (RFLP) recA; APT: automated phenotypic tests; CPT: conventional phenotypic tests; k: kappa index; MALDI-TOF: matrix-assisted laser desorption ionization-time of flight; PCR: polymerase chain reaction; SE: standard error.

TABLE III

A comparative assessment of automated phenotypic tests (APT) and conventional phenotypic tests (CPT) proficiency for Burkholderia cepacia complex (Bcc) identification

\begin{tabular}{lcccc}
\hline Test & $\begin{array}{c}\text { Sensitivity } \\
(\%)\end{array}$ & $\begin{array}{c}\text { Specificity } \\
(\%)\end{array}$ & $\begin{array}{c}\text { PPV } \\
(\%)\end{array}$ & $\begin{array}{c}\text { NPV } \\
(\%)\end{array}$ \\
\hline APT & 97.62 & 100 & 100 & 94.12 \\
CPT & 94.44 & 81.25 & 91.89 & 86.67 \\
\hline
\end{tabular}

analysis performed with 54 isolates presumptively identified as Bcc. NPV: negative predictive value; PPV: positive predictive value.

Antimicrobial susceptibility data showed that the isolates were highly resistant to SUT. Amongst the isolated Bcc species, B. vietnamiensis showed a higher percentage of resistance to all antibiotics than that displayed by the other species identified in our study and these findings contradicted previous findings (Vermis et al. 2003), which showed that B. vietnamiensis was the most sensitive species among the 142 clinical and environmental Bcc samples. Prolonged and aggressive antibiotic therapy is required for treatment of patients with Bcc chronic infection (Leitão et al. 2008). Therefore, antibiotic susceptibility pattern data for local Bcc strains are important because these microorganisms have intrinsic resistance and develop in vivo resistance to several classes of antimicrobials and thereby limit the effectiveness of empirical antibiotic therapy (Drevinek \& Mahenthiralingam 2010). In conclusion, a polyphasic approach that combines biochemical and molecular tests is required to accurate identification of Bcc species infecting CF patients. In the studied population, B. cenocepacia was the prevalent species, predominantly IIIA subtype. Identification by MALDI-TOF and sequencing of rec $A$ agreed in $97.2 \%$ of the isolates. Although MALDI-TOF results completely agree with the results of rec $A$ sequencing for the majority of the strains, it proved not to be able to identify B. cenocepacia subtypes. Between the two phenotypic assays, APT was determined to be a better analytical method than CPT was and can be used in laboratories without molecular assay facilities. However, phenotypic assays were not useful for Bcc species differentiation. B. vietnamiensis was the Bcc species resistant to the higher number of antimicrobials. SUT was the antimicrobial with the highest percentage of resistance. The high degree of antimicrobial susceptibility obtained in our study may be due the fact that the studied strains are from outpatient, possibly undergoing minor antimicrobial selective pressure.

\section{ACKNOWLEDGEMENTS}

To Bruker Corporation of Brazil, for support in the MALDI-TOF.

\section{REFERENCES}

Bittar F, Rolain J 2010. Detection and accurate identification of new or emerging bacteria in cystic fibrosis patients. Clin Microbiol Infect 16: 809-820.

CFC - Cystic Fibrosis Canada 2015. The Canadian cystic fibrosis registry - 2013 annual report. Available from: cysticfibrosis.ca/wpcontent/uploads/2015/02/Canadian-CF-Registry-2013-FINAL.pdf.

CLSI - Clinical and Laboratory Standards Institute 2012. Methods for dilution antimicrobial susceptibility tests for bacteria that grow aerobically. Approved standard, CLSI document M7-A9, 9th ed., CLSI, Wayne, 88 pp.

CLSI - Clinical and Laboratory Standards Institute 2014. Performance standards for antimicrobial susceptibility testing, Twenty-Fourth Informational Supplement, CLSI document M100-S24, Wayne, CLSI, 230 pp.

Coenye T, Vandamme P, Govan JR, LiPuma JJ 2001. Taxonomy and identification of the Burkholderia cepacia complex. J Clin Microbiol 39: 3427-3436.

Dalmastri C, Pirone L, Tabacchioni S, Bevivino A, Chiarini L 2005. Efficacy of species-specific recA PCR tests in the identification of Burkholderia cepacia complex environmental isolates. FEMS Microbiol Lett 246: 39-45.

De Boeck K, Malfroot A, Van Schil L, Lebecque P, Knoop C, Govan JRW, Doherty C, Laevens S, Vandamme P 2004. Epidemiology of Burkholderia cepacia complex colonisation in cystic fibrosis patients. Eur Respir J 23: 851-856. 
Drevinek P, Mahenthiralingam E 2010. Burkholderia cenocepacia in cystic fibrosis: epidemiology and molecular mechanisms of virulence. Clin Microbiol Infect 16: 821-830.

Fehlberg LCC, Andrade LHS, Assis DM, Pereira RHV, Gales AC, Marques EA 2013. Performance of MALDI-ToF MS for species identification of Burkholderia cepacia complex clinical isolates. Diagn Microbiol Infect Dis 77: 126-128.

Gilligan P, Kiska D, Appleman M 2006. Cumitech 43, Cystic fibrosis microbiology, ASM Press, Washington DC, 36 pp.

Henry DA, Campbell ME, LiPuma JJ, Speert DP 1997. Identification of Burkholderia cepacia isolates from patients with cystic fibrosis and use of a simple new selective medium. J Clin Microbiol 35: 614-619.

Khot PD, Couturier MR, Wilson A, Croft A, Fisher MA 2012. Optimization of matrix-assisted laser desorption ionization-time of flight mass spectrometry analysis for bacterial identification. $J$ Clin Microbiol 50: 3845-3852.

Landis JR, Koch GG 1977. The measurement of observer agreement for categorical data. Biometrics 33: 159-174.

Leitão JH, Sousa SA, Cunha MV, Salgado MJ, Melo-Cristino J, Barreto MC, Sá-Correia I 2008. Variation of the antimicrobial susceptibility profiles of Burkholderia cepacia complex clonal isolates obtained from chronically infected cystic fibrosis patients: a five-year survey in the major Portuguese treatment center. Eur $J$ Clin Microbiol Infect Dis 27: 1101-1111.

LiPuma JJ 2010. The changing microbial epidemiology in cystic fibrosis. Clin Microbiol Rev 23: 299-323.

LiPuma JJ, Spilker T, Gill LH, Campbell PW, Liu L, Mahenthiralingam E 2001. Disproportionate distribution of Burkholderia cepacia complex species and transmissibility markers in cystic fibrosis. Am J Respir Crit Care Med 164: 92-96.

Mahenthiralingam E, Baldwin A, Dowson CG 2008. Burkholderia cepacia complex bacteria: opportunistic pathogens with important natural biology. J Appl Microbiol 104: 1539-1551.

Mahenthiralingam E, Baldwin A, Vandamme P 2002. Burkholderia cepacia complex infection in patients with cystic fibrosis. $J$ Med Microbiol 51: 533-538.

Mahenthiralingam E, Bischof J, Byrne S, Radomski C, Davies J, Av-gay Y 2000. DNA-based diagnostic approaches for identification of Burkholderia cepacia complex, Burkholderia vietnamiensis, Burkholderia cepacia genomovars I and III. J Clin Microbiol 38: 3165-3173.

Navrátilová L, Chromá M, Hanulík V, Raclavský V 2013. Possibilities in identification of genomic species of Burkholderia cepacia complex by PCR and RFLP. Pol J Microbiol 62: 373-376.

Peeters C, Zlosnik JEA, Spilker T, Hird TJ, LiPuma JJ, Vandamme P 2013. Burkholderia pseudomultivorans sp. nov., a novel Bur- kholderia cepacia complex species from human respiratory samples and the rhizosphere. Syst Appl Microbiol 36: 483-489.

Schreckenberger P, Daneshvar M, Weyant R, Hollis D 2003. Acinetobacter, Achromobacter, Chryseobacterium, Moraxella and other nonfermentative Gram-negative rods. In P Murray, R Baron, J Jorgensen, M Pfaller, R Yolken (eds.), Manual of clinical microbiology, ASM Press, Washington DC, 1212 pp.

Smet B, Mayo M, Peeters C, Zlosnik JE, Spilker T, Hird TJ, LiPuma JJ, Kidd TJ, Kaestli M, Ginther JL, Wagner DM, Keim P, Bell SC, Jacobs JA, Currie BJ, Vandamme PA 2015. Burkholderia stagnalis sp. nov. and Burkholderia territorii sp. nov., two novel Burkholderia cepacia complex species from environmental and human sources. Int J Syst Evol Microbiol 65: 2265-2271.

Souza HAPHM 2012. Detecção precoce e monitoração da colonização por pseudomonas aeruginosa em crianças com fibrose císti$c a$, PhD Thesis, Universidade Federal do Paraná, Curitiba, 89 pp.

Souza HAPHM, Nogueira KS, Matos AP, Vieira RP, Riedi CA, Rosário NA, Telles FQ, Dalla-Costa LM 2006. Early microbial colonization of cystic fibrosis patients identified by neonatal screening with emphasis on Staphylococcus aureus. J Pediatr (Rio J) 82: 377-382.

Vandamme P, Dawyndt P 2011. Classification and identification of the Burkholderia cepacia complex: past, present, and future. Syst Appl Microbiol 34: 87-95.

Vanlaere E, Baldwin A, Gevers D, Henry D, de Brandt E, LiPuma JJ, Mahenthiralingam E, Speert DP, Dowson C, Vandamme P 2009. Taxon K, a complex within the Burkholderia cepacia complex, comprises at least two novel species, Burkholderia contaminans sp. nov. and Burkholderia lata sp. nov. Int J Syst Evol Microbiol 59: 102-111.

Vanlaere E, LiPuma JJ, Baldwin A, Henry D, de Brandt E, Mahenthiralingam E, Speert D, Dowson C, Vandamme P 2008. Burkholderia latens sp. nov., Burkholderia diffusa sp. nov., Burkholderia arboris sp. nov., Burkholderia seminalis sp. nov., and Burkholderia metallica sp. nov., novel species within the Burkholderia cepacia complex. Int J Syst Evol Microbiol 58: 1580-1590.

Vermis K, Coenye T, Mahenthiralingam E, Nelis HJ, Vandamme P 2002a. Evaluation of species-specific recA-based PCR tests for genomovar level identification within the Burkholderia cepacia complex. J Med Microbiol 51: 937-940.

Vermis K, Vandamme P, Nelis HJ 2003. Burkholderia cepacia complex genomovars: utilization of carbon sources, susceptibility to antimicrobial agents and growth on selective media. J Appl Microbiol 95: 1191-1199.

Vermis K, Vandekerckhove C, Nelis HJ, Vandamme PAR 2002b. Evaluation of restriction fragment length polymorphism analysis of $16 \mathrm{~S}$ rDNA as a tool for genomovar characterisation within the Burkholderia cepacia complex. FEMS Microbiol Lett 214: 1-5. 